Review

\title{
Small role with big impact: miRNAs as communicators in the cross-talk between cancer-associated fibroblasts and cancer cells
}

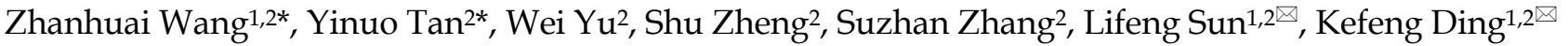 \\ 1. Department of Surgical Oncology, Second Affiliated Hospital of School of Medicine, Zhejiang University, Jiefang Road 88, Hangzhou, Zhejiang Province, \\ 310009, China. \\ 2. The Key Laboratory of Cancer Prevention and Intervention, China National Ministry of Education, Jiefang Road 88, Hangzhou, Zhejiang Province, 310009, \\ China. \\ * Equal contributors. \\ $\triangle$ Corresponding authors: Prof Kefeng Ding, Prof Lifeng Sun. Tel: +86 571 87784820; Fax: +86 571 87784820; Email: Dingkefeng@zju.edu.cn, \\ Sunlifeng@zju.edu.cn.
}

(c) Ivyspring International Publisher. This is an open access article distributed under the terms of the Creative Commons Attribution (CC BY-NC) license (https:// creativecommons.org/licenses/by-nc/4.0/). See http://ivyspring.com/terms for full terms and conditions.

Received: 2016.09.23; Accepted: 2016.12.09; Published: 2017.02.25

\begin{abstract}
Cancer microenvironment is composed of numerous components that can support cancer cell proliferation, promote cancer progression and contribute to cancer treatment resistance. The major components of caner microenvironment are fibroblasts, endothelial cells, immune cells as well as cytokines, chemokines, and extracellular matrix (ECM) all of which surround tumor cells as the core and cross talk with each other. Among them, cancer-associated fibroblasts (CAFs) play an important role in promoting cancer progression by secreting various pro-inflammatory factors. MicroRNAs (miRNAs) are small noncoding RNAs that negatively regulate protein expression both in cancer cell and normal stromal cells. Changes of miRNAs expression in cancer-associated fibroblasts can be induced both by cancer cells and other stromal cells. This change can arise through direct interaction or by secreted paracrine factors or even by secreted miRNAs. The desregulated miRNAs in cancer-associated fibroblasts then enhance the CAFs phenotype and assist their cancer promotion ability. Explore the regulatory function of miRNAs in the complex communication between cancer cells and cancer microenvironment is important to understand the process of tumor progression and may help to develop new therapeutic strategies. This review provides an updated content of latest research advances about the relevance of miRNAs in the interaction between cancer cells and the CAFs. We will describe miRNAs which are differential expressed by NFs and CAFs, their function in regulating fibroblasts activation as well as miRNAs expressed in CAFs as prognostic factors in cancer stroma in recent studies. We will also discuss miRNA as an important player in CAFs mediated regulation of cancer progression and metastasis, cancer metabolism, cancer stem cell property and chemoresistance.
\end{abstract}

Key words: MiRNAs; Cancer associated fibroblasts; Cancer Microenvironment

\section{Introduction}

Fibroblasts are the most common components of the connective tissue. In wound healing process, the phenotype of fibroblasts is transformed into an activated state. The activated fibroblasts have certain characteristics both like fibroblasts and smooth muscle cells. In many cases, tumors are commonly analogized as wounds that do not heal. The reconstruction process of stromal cells launched by cancer cells share some common similarities with wound healings. Activated fibroblasts also exist in cancer, where they are so-called cancer-associated fibroblasts (CAFs). CAFs are distinct from normal fibroblasts which own certain markers, enhanced pro-tumorigenic properties and can produce a variety 
of pro-inflammatory factors. (1-3) CAFs can even recruit other stromal cell types to the primary lesion and the metastatic lesions of cancer. They all participate in the construction of the cancer microenvironment to promote tumor proliferation, invasion and metastasis. (4). The exact origin of CAFs and the mechanisms that normal cell become CAFs are still not clear, but current evidence indicates that a considerable part of the CAFs are originated from local normal fibroblasts surround cancer cells by continuous contact with them. (5) Multiple signal axis of CAFs appeared to be abnormally activated compared with normal fibroblasts including NF- $\mathrm{kB}$ signaling, IL-6/STAT3 signaling, FGF-2/ FGFR1 signaling and TGF- $\beta$ / SMAD signaling. (6-8). It is surprised that primary cultured CAFs may sustain their phenotype over several passages in vitro. This indicates CAFs may undergo genetic or epigenetic alterations. $(1,2)$.

MiRNAs are a class of small non-coding RNA which functioned as the main participants of posttranscriptional gene regulation. They function in both normal physiological and pathological condition. Numerous studies have demonstrated that miRNAs can act as tumor suppressors or promoters regulating the behaviors of tumor in addition to various proteins encoding genes $(9,10)$. MiRNAs is able to regulate cancer biological behavior at many aspects such as cancer proliferation and metastasis, cancer immune escape, cancer cell immortalization, cancer related inflammation, cancer stem cell property, cancer metabolism, cancer angiogenesis (11). As malignant tumors have increasingly been recognized as a complex pathological organs, the specialized cell types within tumor microenvironment should be individually studied so as to better understand cancer biology (12). Previous studies have shown that cancer cells modulate stromal cells that lack genomic instabilities in the cancer microenvironment by changing their miRNAs expression profiles $(13,14)$. In addition, circulating miRNAs have been found as specific biomarkers for both early detection of carcinogenesis and prediction of treatment response. Accumulating evidences showed that tumor cells communicate with stromal cells in the local environment or even in the remote organs via secretion microvesicles packed with miRNAs. These miRNAs then released from microvesicles into the target stromal cells as messengers to dictate them so as to facilitate tumor progression and metastasis. (15)

Recently, a few studies show the genetic changes in CAFs $(16,17)$. However, deep analysis of CAFs from breast and ovarian cancer reals genetic alterations in CAFs are actually uncommon or even rare $(18,19)$. Genomewide analysis of breast cancer stroma suggests that the stable phenotype of CAFs is likely to be regulate by epigenetic alterations (16). In recent studies, miRNAs desregulation has been detected in other cancer-associated stromal cell types, such as cancer-associated macrophages (TAMs), osteoclasts and myeloid derived suppressor cells (MDSCs). Studies also suggest the differential miRNAs expression pattern in CAFs and normal fibroblast (NFs) from several types of cancers and propose that miRNAs dysregulation is associated with various CAFs behavior. $(9,20)$ This review will describe miRNAs which are differential expressed between NFs and CAFs, their function in regulating fibroblasts activation as well as miRNAs expressed by CAFs as the prognostic factors in cancer. We will also discuss role of miRNAs in CAFs mediate cancer progression and metastasis, cancer metabolic changes, cancer stem cell property and cancer chemoresistance. (Fig 1) The main findings of miRNAs dysregulation contribute to CAFs phenotype and functions are summarized in Supplementary Table 1.

\section{Interaction between CAFs and cancer cells by secreted miRNAs}

Extracellular vesicles (EVs) are novel identified vectors for the intercellular communication. In general, EVs are defined as a series of membrane-bound organelles between 40 and 1,000 $\mathrm{nm}$ in diameter. Among these EVs, exosomes are derived from the endosomal compartment with 30-100 nm diameters. Multiple components of tumor microenvironment including cancer cells, CAFs and inflammatory cells can communicate by secreting EVs. The secreted EVs can not only affect the surrounding cells but also travel to the distant organs remodeling the pre-metastatic microenvironment. The essential massages contained in EVs from the secreted cell include proteins, nucleic acids and lipids. $(21,22)$. Morello et al show that miR-1227 is a miRNA abundant in the large oncosomes produced by RWPE-2 tumorigenic prostate cells. MiR-1227 can transfer into CAFs by Large oncosomes, and enhance CAFs migration (23). MiR-409 is found to up regulate in CAFs from prostate cancer. MiR-409 transfected normal prostate fibroblasts acquire CAFs phenotype and secrete miR-409 via EVs to induce cancer cell EMT process in vitro and in vivo. (24) Apart from transferred by excellular vesicles like exosome, miRNAs can also be released directly into the extracellular fluid and uptake by other components of tumor microenvironment and exert their effects. MiR-133b was found to increase both in the conditioned media and exosome from prostate patient-derived CAFs compare to these obtained from normal fibroblasts. The miR-133b then acts as soluble 
factor for paracrine stimulation of both fibroblasts and tumor cells and further increases their endogenous miR-133b expression. Thus, a positive feedback loop of secreted miR-133b in both prostate cancer cell and CAFs is formed (25).

\section{MiRNAs dysregulation contribute to CAFs phenotype}

According to Shalom Madar, 'CAFs' should be defined as a fibroblast dynamic state in the presence of cancer microenvironment that possess pro-tumorigenic properties - for brevity a 'CAF state'(26).Currently, the most reliable methods to define CAFs in vitro is a combination of morphological feature and biological marker (7). CAFs appear morphologically in a form of activated fibroblasts and produce massive ECM ingredients. Typical activated fibroblasts are different from normal fibroblasts which comprised of abundant rough endoplasmic reticulum and a large oval euchromatic nucleus with one or two nucleoli. The inactive normal fibroblasts comprise smaller endoplasmic reticulum, flattened and heterochromatic nucleus. (3). Several proteins have been used as markers of CAFs including a-SMA, tenascin- $\mathrm{C}$, neuron glial antigen
(NG2), PDGFR - $\alpha / \beta$, FAP, thy-1 (CD90), podoplanin, CAV-1 and FSP-1/S100A4. Among them, a-SMA is the most commonly used marker. $(27,28)$ In order to sustain the 'CAF state' in tumor microenvirionment, three possible mechanisms may involve in fibroblasts activation: genetic mutations, epigenetic alterations, and persistent environmental effects. Among them, changes of miRNAs expression which belong to epigenetic alterations are considered as an important controller in maintain 'CAF state' $(26,29)$. MiRNAs involved in fibroblasts activation are heterogeneous among organs and tumor types, this may partly due to the phenotypic and functional heterogeneity in CAFs from different tissue and tumor type. Recently, miRNAs were found to release from CAFs or cancer cells into the media and act as a paracrine stimulus to activate the adjacent fibroblasts, thus facilitate spreading 'CAF state' into the whole surrounding fibroblasts in cancer if necessary (25). Several studies indicated that exogenous overexpress of miRNAs is able to convert normal fibroblasts into CAFs-like cells with the same phenotype in vitro (Table 1). These evidences confirmed the critical role of miRNAs in the process of fibroblasts activation.

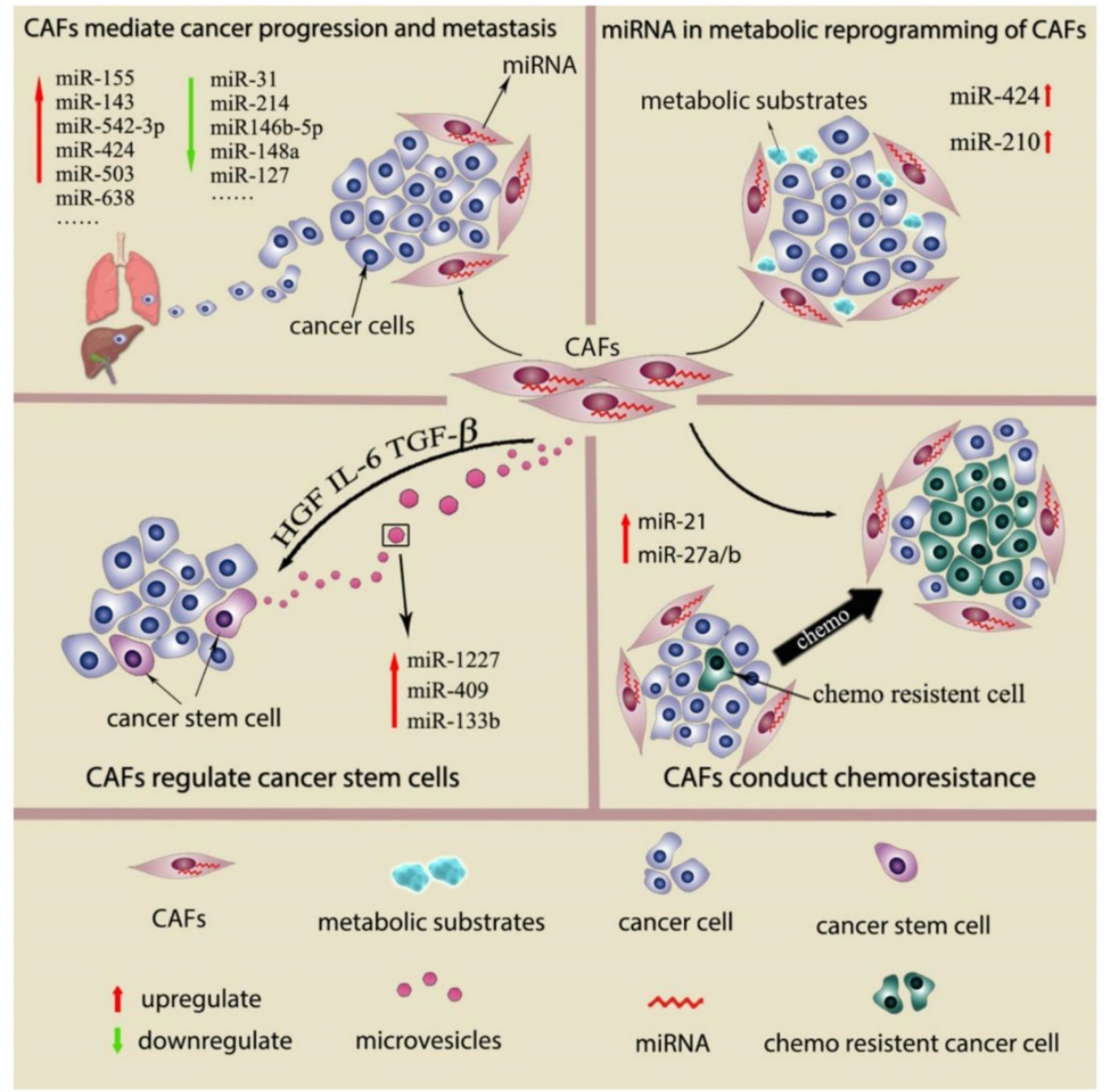

Figure 1. miRNAs dysregulation contributes to CAFs phenotype and functions 
Table 1. MiRNAs involved in fibroblasts activation

\begin{tabular}{|c|c|c|c|c|c|}
\hline Ref & Fibroblasts source & $\begin{array}{l}\text { Differential } \\
\text { expression } \\
\text { MicroRNAs }\end{array}$ & $\begin{array}{l}\text { Up-regulate/down-regulate in } \\
\text { CAFs }\end{array}$ & methods to verify microRNA function & CAFs markers \\
\hline \multirow[t]{3}{*}{ (25) } & $\begin{array}{l}\text { human prostate } \\
\text { cancer tissue }\end{array}$ & miR-133b & Up-regulate & $\begin{array}{l}\text { Exogenous Overexpression of the } \\
\text { miR-133b in primary normal prostate } \\
\text { firoblasts }\end{array}$ & $\begin{array}{l}\text { a-SMA, FAP, } \\
\text { S100A4 }\end{array}$ \\
\hline & TGF $\beta$ induced HPFs & & & & \\
\hline & IL-6 induced HPFs & & & & \\
\hline \multirow[t]{3}{*}{$(40)$} & breast cancer tissue & miR-146b-5p & down-regulate & $\begin{array}{l}\text { ectopic expression of miR-146b-5p in } \\
\text { CAFs }\end{array}$ & a-SMA \\
\hline & normal breast tissue & & & $\begin{array}{l}\text { inhibition of miR-146b-5p in breast } \\
\text { stromal fibroblasts }\end{array}$ & \\
\hline & $\begin{array}{l}\text { normal breast } \\
\text { ajacent to cancer }\end{array}$ & & & & \\
\hline$(69)$ & $\begin{array}{l}\text { mouse pancreat } \\
\text { tissue }\end{array}$ & miRNA-155 & Up-regulate & $\begin{array}{l}\text { treated with mouse pancreat normal } \\
\text { fibroblasts with pacreatic cancer cell } \\
\text { derived microvesicles containing } \\
\text { miRNA-155 }\end{array}$ & a-SMA and FAP \\
\hline \multirow[t]{2}{*}{$(38)$} & gastric cancer tissue & $\operatorname{miR}-149$ & down-regulate & ectopic expression of miR-149 in CAFs & FAP \\
\hline & gastric tissue & & & inhibition of miR-149 in NFs & \\
\hline \multirow[t]{2}{*}{$(66)$} & esophageal cancer & $\operatorname{miR}-27 \mathrm{a} / \mathrm{b}$ & Up-regulate & $\begin{array}{l}\text { Normal fibroblasts from esophagus } \\
\text { transfected with pre-miR-27a or } \\
\text { pre-miR-27b }\end{array}$ & $\begin{array}{l}\text { a-SMA and } \\
\text { vimentin }\end{array}$ \\
\hline & $\begin{array}{l}\text { normal esophageal } \\
\text { tissue }\end{array}$ & & & & \\
\hline \multirow[t]{2}{*}{$(24)$} & prostate cancer & miR-409 & Up-regulate & $\begin{array}{l}\text { Ectopic expression of miR-409 in normal } \\
\text { prostate stromal fibroblasts }\end{array}$ & a-SMA \\
\hline & $\begin{array}{l}\text { normal prostate } \\
\text { tissue }\end{array}$ & & & & \\
\hline \multirow[t]{2}{*}{$(70)$} & $\begin{array}{l}\text { scirrhous type } \\
\text { gastric cancer }\end{array}$ & $\operatorname{miR}-145$ & Up-regulate & $\begin{array}{l}\text { transfected normal fibroblasts with } \\
\text { pre-miR-145 and inhibit miR-145 } \\
\text { expression in CAFs }\end{array}$ & a-SMA \\
\hline & $\begin{array}{l}\text { normal gastric } \\
\text { tissue }\end{array}$ & & & & \\
\hline \multirow[t]{2}{*}{$(71)$} & esophageal cancer & $\operatorname{miR}-21$ & Up-regulate & $\begin{array}{l}\text { HGF-1 fibroblasts transfected or inhibited } \\
\text { with miR-21 }\end{array}$ & S100A4 \\
\hline & HGF-1cell & & & & \\
\hline \multirow[t]{2}{*}{$(43)$} & $\begin{array}{l}\text { MRC5 lung } \\
\text { fibroblast cell }\end{array}$ & & Up-regulate & $\begin{array}{l}\text { Stable ectopic expression of miR-21 in } \\
\text { immortalised MRC } 5 \text { fibroblasts }\end{array}$ & a-SMA \\
\hline & $\begin{array}{l}\text { Primary colonic } \\
\text { fibroblasts }\end{array}$ & & & & \\
\hline (39) & $\begin{array}{l}\text { benign prostatic } \\
\text { hyperplasia }\end{array}$ & $\operatorname{miR}-210$ & Up-regulate & $\begin{array}{l}\text { Exogenous Overexpression of the } \\
\text { miR-133b in primary normal prostate } \\
\text { firoblasts }\end{array}$ & $\begin{array}{l}\text { a-SMA and } \\
\text { collagen I }\end{array}$ \\
\hline
\end{tabular}

\section{MiRNAs in CAFs as stromal marker for cancer prognosis}

In addition to the functional researches that demonstrate the cancer supportive effect of cancer stroma, several studies also try to confirm the clinical and pathological importance of cancer stroma in cancer development $(30,31)$. It is hypothesized that if certain components from cancer stroma are related to the tumor-supportive capacity, they may also become promising cancer prognostic factors. As the cancer promotion abilities of CAFs were increasingly been recognized, many strategies were conducted to explore the prognostic value of CAFs. FAP, a marker of activated fibroblasts during tissue remodeling and CAFs, is demonstrated a relation to shorter survival in a small study of unselected NSCLC patients (32). Podoplanin, a membrane glycoprotein which is suggested as a novel marker for CAFs, is demonstrated to be correlated to shorter recurrence free survival in early stage lung cancer. $(33,34)$ However, in breast cancer, FAP expression in CAFs showed significantly longer disease free and overall survival (35) and podoplanin expression in CAFs expression yielded conflicting results regarding its prognostic value $(36,37)$. Thus, there is still no conclusion about if the activate fibroblasts marker are negative prognostic factor of cancer. It is possible that CAFs from different cancers express the same markers have different properties due to the large heterogeneity between CAFs (28). Therefore, due to its complex signaling pathway and the diversity of cells origin, it is still challenging to determine 
common CAFs markers which are strongly related to the clinical features. Based on differential miRNAs expression profile between normal and cancer associated fibroblasts, several single miRNAs were chosen to verify their prognostic value. (Table 2) However, these studies were in the light of a single miRNAs marker just providing a reference value of the candidates and did not come to the prognostic results based on multivariate analysis. As single miRNAs markers are insufficient to describe the heterogeneous subgroups of CAFs, developing a comprehensive miRNAs signature seems to better define 'CAFs state' and subsequently for prognostication. Future studies are likely to integrate global miRNAs expression profiling information of CAFs from publicly available data sets and identify miRNAs signatures capturing different aspects of CAFs which associated with patient outcome for potential clinical application.

Table 2. MiRNA in CAFs as prognosis factor in cancer stroma

\begin{tabular}{|c|c|c|c|c|c|c|}
\hline Ref & $\begin{array}{l}\text { Cancer } \\
\text { type }\end{array}$ & MicroRNA & $\begin{array}{l}\text { Up } \\
\text { regulate/ } \\
\text { down } \\
\text { regulate } \\
\text { in CAFs }\end{array}$ & methods & $\begin{array}{l}\text { Survival } \\
\text { analysis } \\
\text { Kaplan-Meier }\end{array}$ & $\begin{array}{l}\text { Case } \\
\text { number }\end{array}$ \\
\hline (72) & $\begin{array}{l}\text { Gastric } \\
\text { cancer }\end{array}$ & $\begin{array}{l}\text { miRNA-106 } \\
\mathrm{b}\end{array}$ & $\begin{array}{l}\text { Up } \\
\text { regulate }\end{array}$ & ISH & Poor survival & 120 \\
\hline (46) & $\begin{array}{l}\text { Gastric } \\
\text { cancer }\end{array}$ & miR-143 & $\begin{array}{l}\text { Up } \\
\text { regulate }\end{array}$ & $\begin{array}{l}\text { Cytological } \\
\text { localization } \\
\text { qRT-PCR }\end{array}$ & $\begin{array}{l}\text { worse } \\
\text { cancer-specific } \\
\text { mortality }\end{array}$ & 68 \\
\hline (24) & $\begin{array}{l}\text { Prostate } \\
\text { cancer }\end{array}$ & miR-409 & $\begin{array}{l}\text { Up } \\
\text { regulate }\end{array}$ & ISH & $\begin{array}{l}\text { correlated with } \\
\text { higher Gleason } \\
\text { score and poor } \\
\text { survival }\end{array}$ & 55 \\
\hline (70) & $\begin{array}{l}\text { Gastric } \\
\text { cancer }\end{array}$ & miR-145 & $\begin{array}{l}\text { Up } \\
\text { regulate }\end{array}$ & qRT-PCR & $\begin{array}{l}\text { worse } \\
\text { cancer-specific } \\
\text { mortality }\end{array}$ & 71 \\
\hline
\end{tabular}

\section{Role of miRNAs in CAFs mediated cancer progression and metastasis}

Compared with normal fibroblasts, primary cultured CAFs derived from malignances have an enhanced proliferate ability and produce more growth factors, collagen and ECM regulators. This was so called the intrinsic CAF phenotype. Apart from the intrinsic phenotype, the extrinsic phenotype of CAFs is the cancer promotion capability, which is also the gold standard for identifying CAFs. This effect is in large part driven by the secretion of cytokines and growth factors that affect proliferation, survival, adhesion, and migration of cancer cells and is usually confirmed by coculture test in vitro and in vivo. (26) In the light of differential miRNAs expression pattern between CAFs and NFs, studies were carried out to determine key functions of miRNAs that play important role in CAFs. By genetic intervention of miRNAs expression in NFs, NFs can not only acquire activated fibroblasts marker (Table 1) but also exhibited tumor enhancing abilities. Meanwhile, transform the expression of dysregulated miRNAs in CAFs attenuated their tumor enhancing functions.

One of the classic cases that described miRNAs regulation of fibroblasts tumor promotion abilities is conducted in ovarian cancer. The study suggested that normal ovarian fibroblasts can be reprogramed into CAFs by combined the upregulating miR-155 and downregulating of miR-214 and miR-31. The enhanced tumor-promoting functions by three miRNAs intervention in fibroblasts was validate in a 3 dimension co-culture model in vitro. MiR-214 both regulates fibroblasts migration and tumor cells invasion, miR-155 mainly affects the invasion ability of tumor cells, and the function of miR-155 is related to the colony formation of tumor cells. This study also identified CCL5 as a target of miR-214 and showed anti-CCL5 abolished the tumor promotion ability of miR-214 transfected CAFs.

Epithelial-to-mesenchymal transition (EMT) is a critical step in cancer metastasis, and this procedure can be induced by CAFs. In gastric cancer, CAFs enhance EMT of cancer cells in a miR-149-IL-6-dependent manner which was verified by EMT marker such as $\mathrm{N}$-cadherin, vimentin and snail (38). When PC3 prostate cancer cells are co-culturing with miR-210 transfected sencent prostate fibroblasts, their EMT traits are enhanced with raised vimentin, ZEB1 and ZEB2 expression and reduced E-cadherin expression (39) .Conditioned media from miR-146-5p-defective breast stromal fibroblasts promote EMT of breast cancer cells through increased N-cadherin, vimentin and Snail expression and decreased E-cadherin expression compared to control cells. (40)

MiRNAs regulate the tumor promoting effects of CAFs through various cytokines and signal pathways such as MMPs, IL-6, transforming growth factor-b/ SMAD signaling, FGF-2/ FGFR1 axis, PTEN signaling, estrogen receptor signaling. Matrix metalloproteinases (MMPs), matrix metalloproteinase inhibitors, and extracellular matrix (ECM) component were commonly secreted by CAFs to regulates other components in tumor microenvironment. (41). It is known that RECK can modulate MMP2 expression through a series of post-transcription mechanism and are widely involved other cancer related biological pathways. (42) Ectopic miR-21 expression in fibroblasts induce myofibroblast transdifferentiation by increased a-SMA expression and resulting in downregulated RECK expression which lead to increased MMP2 activity. (43) Interleukin-6 (IL-6) is a 
multifunctional cytokine which plays key roles in the interaction between tumor cancer and tumor microenvironment (44). In breast cancer, IL-6 is suggested to involve in cancer cell dependent activation of stromal fibroblasts (22). MiR-146b-5p, a cancer suppressor miRNAs which represses various pro-carcinogenic processes, was shown to repress breast stromal fibroblasts activation via IL-6/STAT3 signaling. Ectopic expression of pre-miR146b-5p in breast stromal fibroblasts suppressed their pro-tumorigenic effects by inhibiting their migration/ invasion abilities and their expression of a-SMA, TGF- $\beta 1$ and SDF1. (40) TGF- $\beta$ is also a multifunctional cytokine which important roles in the cross talk between cancer cells and CAFs. The classic TGF- $\beta$ signaling pathway is delivered by the downstream SMAD protein family (45). In scirrhous type gastric cancer, TGF- $\beta$ regulates the expression of collagen type III both in NFs and CAFs in a SMAD-dependent manner. Moreover, miR-143 was identified as a novel regulator of collagens which enhanced collagen type III expression in NFs and CAFs through activation of TGF- $\beta$ / SMAD signaling. Down regulation of miR-143 in fibroblasts significantly abolish TGF- $\beta$ induced collagen type III expression by targeting SMAD2. (46). FGF-2/ FGFR1 axis also mediated the tumor microenvironment cross-talks. Analysis of the FGF signaling in a variety of solid tumors shows that excessive activation of the FGF signaling pathway could promote tumor proliferation, invasion, and drug resistance. (47-52) In prostate cancer, miR-15 and miR-16 were found to be down regulated in CAFs. Upregulation of miR-15 and miR-16 impaired CAFs pro-tumorigenic ability partially by reduced FGF-2 and FGFR1 protein expression. In a mouse model, DU145 prostate cancer cell co-inoculated with a higher amount of miR-15/16 transducted fibroblasts could not even form tumors, whereas empty vector fibroblasts mixed with DU145 strongly promoted tumor formation. A reduced FGF-2 expression in both tumor and stroma part of mouse tumor mass were considered as the possible mechanism (53). PTEN is known as a tumor suppressor gene, while the function of PTEN in CAFs is not well-studied. Pten-miR-320-Ets2 tumor suppressor axis in mammary stromal fibroblasts can modulate the inter-cellular communication within the tumor microenvironment. Loss of Pten in mammary stromal fibroblasts lead to down-regulation of miR-320 and reprogramming the phenotype of neighboring endothelial and cancer cells of the mammary gland by producing tumor promoting secretom in fibroblasts conditioned media. (54) In estrogen-related malignances, estrogens can influence the proliferation, differentiation and biological behavior of tumor by binding to the estrogen receptor (54). Recent studies have suggested that $G$ protein receptors, GPER (G protein estrogen receptor) and GPR30 (G protein-coupled receptor 30 ), are involved in estrogen related effects in many normal and tumor tissues. (55) (21). In breast cancer, it was demonstrated that GPER/miR144/Runx1 signaling pathway exits in CAFs, which play promotion effects through interaction with cancer cells. (56)

\section{Role of miRNAs in CAFs regulation of cancer stem cells}

Currently evidences indicate that $\mathrm{CAFs}$ are participated in cancer stem cell (CSC) regulation by CAFs derived cytokines and chemokines which activate Wnt, Notch, BMPs, hedgehog signaling. CAF modulate of CSCs in the following ways: Firstly, CAFs can directly act on CSCs to induce their self-renew process(57). Secondly, CAFs can reprogram and induce a stem cell phenotype in relatively differentiated tumor cells, thus increasing the CSC pool(58). Thirdly, CAF can stimulate the autocrine signaling loops in CSCs and keep them in a stem cell state. (59) In gastric cancer, MiR-149 was down regulate in CAFs from gastric cancer and high expressed in NFs from adjacent gastric tissue. The ALDH high expression subgroup of gastric cancer cells can be increased by CAFs in a miR-149-IL-6-dependent manner. Supernatant from miR-149 transfected CAFs fail to increase ALDH high expression subgroup cancer cells and this can be rescued by IL-6 supplementation. In contrast, Supernatant from anti-miR-149 transfected NFs obtain the ability to raise ALDH high expression cancer cells like CAFs, and this is abolished by IL-6 neutralizing antibody. As ALDH high expression cancer cell subgroup partly represent the CSCs, the results indicated miR-149 in gastric fibroblasts has a indirect effect on CSC proliferation and CSC phenotype induction. (38)

\section{Role of miRNAs in metabolic reprogramming of CAFs}

Recently, CAFs are found to secrete large amounts of metabolic products including lactate and ketone bodies. These products are then uptake by cancer cells for anabolic metabolism or oxidative phosphorylation (60). Apart from lactate and ketone bodies, a large number of mitochondria metabolic substrates including fatty acids, glutamine, other amino acids are also provided by CAFs to support cancer cells growth(6). In fact, the glycolytic behavior of CAFs has been regarded as a common nature or even as an essential step for CAFs activation, while 
the underlying signaling cascade has been increasingly recognized (61). Metabolic collaboration between catabolic fibroblasts and anabolic tumor cells is detected in several types of malignancies such as breast cancer, head \& neck cancer, prostate cancer and lymphomas. Such catabolic status detected in CAFs is secondary to metabolic stress response induced by ROS. HIF1- $\alpha$ induced aberrant activation of NF-KB signaling in CAFs play key roles in motivate the oxidative stress, autophagy, glycolysis and senescence process.(6).

Several studies reported miRNAs are involved in metabolic reprogramming of CAFs. MiR-210 is a hypoxia-associated miRNAs. When human prostatic fibroblasts were cultured in hypoxic condition, the miR-210 expression was increased by six-fold. MiR-210 transfected prostate fibroblasts induce cell senescence and increase the CAFs marker a-SMA expression. Moreover, senescent fibroblasts induced by miR-210 transfection can supply cancer cells with amounts of L-lactate and ketone bodies. (39) Recent studies suggested that HK2, an isozyme contributes to aerobic glycolysis, is increased in the CAF cells. HK2 expression can be regulated by miRNAs, either directly or indirectly $(62,63)$. MiR-182 targets the 3'UTR of HK2, and regulate HK2 expression in both mRNA and protein level in CAFs. (64) Many rate-limiting enzymes in tricarboxylic acid cycle aerobic metabolism and anaerobic glycolysis are regulated by miRNAs. IDH3a (isocitrate dehydrogenase 3 ) is a critical rate-limiting enzyme in the TCA cycle which is commonly decrease in CAFs. IDH3a downregulation triggers the metabolic switch from oxidative phosphorylation to glycolysis in CAFs, while IDH3a overexpression decreased the expression of glycolysis-related enzymes, including GLUT1, HK2, and PFKM. D. Zhang et al reported that miR-424 is responsible for TGF- $\beta$-induced IDH3a downregulation in CAFs. MiR-424 overexpression increased the expression of glycolysis-related enzymes, including GLUT1, HK2, and PFKM. Lactate production was upregulated in miR-424-overexpressing fibroblasts by approximately 2 -fold compared to control fibroblasts. Glucose uptake was increased more than 2-fold in miR-424-overexpressing fibroblasts compared to control fibroblasts. MiR-424 overexpression also decreased oxygen consumption and oxidative phosphorylation. MiR-424 overexpression increased, whereas miR-424 knockdown decreased HIF-1a in protein level, which is an important player in the regulation of glycolysis. (20)

\section{Role of miRNAs in CAFs conducted chemoresistance}

Chemoresistance is the one of the main cause of poor prognosis in cancer patients. Chemoresistance is not only conducted by cancer cells but also by changes of the components of cancer microenvironment. Among these components, CAFs is a important regulators the sensitivity to chemotherapy. (7) By using a coculture system in vitro, CAF mediated chemoresistance can be detected as a common phenomenon. CAFs secret hepatocyte growth factor (HGF) can lead to target therapy resistance in BRAF-mutant melanoma through mitogen-activated protein kinase (MAPK) signaling pathways (45). Moreover, exosomes derived by CAFs can induce colon cancer cells resistant to 5-fluorouracil (5-FU) or oxaliplatin by increasing the population of cancer stem cells $(7,65)$. Tanaka et al detected in esophageal cancer that patient with high levels of miR-27a/b in serum is correlated with poor chemotherapy response. MiR-27a/b transfected cancer cell shows no difference in chemosensitivity. By contrast, conditioned media from normal fibroblasts transfected with miR-27a/b can reduce the chemosensitivity of esophageal cancer cells to cisplatin in vitro. Increased TGF- $\beta$ level is found in conditioned media from normal fibroblasts transfected with miR-27a/b and administration of TGF- $\beta$ neutralizing antibody to the supernatant recovered cancer cell chemosensitivity (66). Donahue et al conducted a retrospective study to evaluate tissue miR-21 expression levels in predicting gemcitabine or 5 -fluorouracil $(5-\mathrm{FU})$ response in a cohort of pancreatic ductal adenocarcinoma (PDAC) patients from the RTOG 9704 trial. They show that miR-21 is strongly expressed in CAFs in 152/181 $(84 \%)$ patients and miR-21 expression in CAFs is associated with decreased OS in PDAC patients who received 5-FU, but not gemcitabine. The study suggests stromal miR-21 as a marker to guide chemotherapy choice in PDAC patients. (67) Another study shows miR-21 is involved in CAFs related chemoresistance in CRC cancer. Ectopic expression of miR-21 in a human fibroblast cell line shows similar morphological appearance as myofibroblasts with increased a-SMA expression. Moreover, conditioned medium from miR-21-overexpressing fibroblasts protected CRC cells from oxaliplatin-induced apoptosis and increased their proliferative capacity (43). In ovarian cancer, high level of miR-21 is detected in the exosomes from CAFs compared to normal ovary fibroblasts. Exosome miR-21 transferred from CAFs into cancer cell not only promote cell invasion but also suppresses cell apoptosis and 
induced paclitaxel resistance by binding to APAF1.(44) J. Li, et al show that CAFs from lung cancer patient promoted lung cancer cell resistant to cisplatin in a paracrine manner. High amount of SDF-1 is detected in CAFs which facilitated drug resistance and miR-1 was confirmed negatively regulated the expression level of SDF-1 in the CAFs (68).

\section{Future direction}

This review described the cross talk manners between cancer cells and CAFs bridged by miRNAs. The process that normal stromal fibroblasts remodeled by cancer cell into CAFs or 'CAFs state' cells is reflected by changes of miRNAs expression profile. These changed miRNAs then directly or indirectly participate in multiple functions of CAFs as a response. Due to the different biological characteristics between cancer cells and CAFs, the same miRNAs may play different roles in them. Meanwhile, although there are some special miRNAs deregulations in CAFs from certain kind of cancers, some miRNAs are shown to play a common role in CAFs in different tumors. These miRNAs may have a more basic function in the various communications between cancer cells and CAFs. The studies in this review indicated that miRNAs are involved in almost all the aspects of the communication between cancer cells and CAFs including CAFs regulation of cancer progression and metastasis, cancer stem cell property, chemoresistance as well as cancer metabolism. The novel cell communication manner by intracellular exosome miRNAs transition provides us a new insight to explore the complicated network of the tumor microenvironment. As our understanding of miRNAs biology and cell-cell communication improved, the importance of miRNAs in CAFs regulation network will be further explored. The powerful ability to promote tumor progression and relatively stable genetic background makes CAFs a considerable potential targets for cancer therapy. MiRNAs regulation increase the difficulty to understand the complex interactions between cancer cell and CAFs, however, it also provide a new opportunity for treatment. Future studies should identify which of these desregulated miRNAs in CAFs are passengers and which ones are drivers in CAFs network for development of miRNAs target therapy. Meanwhile, developing effective agents to block the extracellular miRNAs travelling across CAFs and cancer cells shall be a promising anti-cancer method. In conclusion, the comprehensive miRNAs regulation networks involved in the cross talk between cancer cell and CAFs is worth deep investigation and may yield novel cancer treatment solutions that assist the current cancer management to achieve a better response.

\section{Supplementary Material}

Supplementary table 1.

http://www.ijbs.com/v13p0339s1.pdf

\section{Acknowledgements}

This work was supported by grants from the National Natural Science Foundation of China (No. 81272455, 81472664), the Public Welfare in Health Industry, 2014, Health Ministry of China (NO. 201402015) and Key Projects in the National Science \& Technology Pillar Program during the Twelfth Five-year Plan Period (No. 2014BAI09B07). The sponsors of the study had no role in study design, data collection, data analysis, results interpretation, writing the paper and the decision to submit the paper for publication.

\section{Abbreviations}

5-FU, 5-fluorouracil; CAFs, cancer-associated fibroblasts; CM, conditioned media; COX-2, cyclooxygenase-2; E2, estrogen ER, estrogen receptor; EMT, Epithelial-Mesenchymal Transition; FGF, fibroblast growth factor; GC, gastric cancer; GPER, hMAPK, hyperactive MAPK signaling; HK2, Hexokinase 2; IDH3a, isocitrate dehydrogenase 3a; IL, interleukin; MAPK, mitogen activated protein kinase; miRNA, microRNA; NFs, normal fibroblasts; HPFs, human prostate fibroblasts; H.P., $\mathrm{H}$ pyloriinfection; OS, overall survival; $\mathrm{PaC}$, pancreatic cancer; PDAC, pancreatic ductal carcinoma; PGE2, prostaglandin E2; TGF, transforming growth factor; a-SMA, alpha-smooth muscle actin; SDF-1, stromal cell-derived factor 1 .

\section{Competing Interests}

The authors have declared that no competing interest exists.

\section{References}

1. Orimo A, Gupta PB, Sgroi DC, Arenzana-Seisdedos F, Delaunay T, Naeem R, Carey VJ, et al. Stromal fibroblasts present in invasive human breast carcinomas promote tumor growth and angiogenesis through elevated SDF-1/CXCL12 secretion. Cell 2005;121:335-348.

2. Erez N, Truitt M, Olson P, Arron ST, Hanahan D. Cancer-Associated Fibroblasts Are Activated in Incipient Neoplasia to Orchestrate Tumor-Promoting Inflammation in an NF-kappaB-Dependent Manner. Cancer Cell 2010;17:135-147.

3. Kalluri R, Zeisberg M. Fibroblasts in cancer. Nat Rev Cancer 2006;6:392-401.

4. Psaila B, Lyden D. The metastatic niche: adapting the foreign soil. Nat Rev Cancer 2009;9:285-293.

5. Chou J, Shahi P, Werb Z. microRNA-mediated regulation of the tumor microenvironment. Cell Cycle 2013;12:3262-3271.

6. Martinez-Outschoorn UE, Lisanti MP, Sotgia F. Catabolic cancer-associated fibroblasts transfer energy and biomass to anabolic cancer cells, fueling tumor growth. Semin Cancer Biol 2014;25:47-60.

7. Ishii G, Ochiai A, Neri S. Phenotypic and functional heterogeneity of cancer-associated fibroblast within the tumor microenvironment. Adv Drug Deliv Rev 2015. 
8. Gandellini P, Andriani F, Merlino G, D'Aiuto F, Roz L, Callari M. Complexity in the tumour microenvironment: Cancer associated fibroblast gene expression patterns identify both common and unique features of tumour-stroma crosstalk across cancer types. Semin Cancer Biol 2015.

9. Suzuki HI, Katsura A, Matsuyama H, Miyazono K. MicroRNA regulons in tumor microenvironment. Oncogene 2015;34:3085-3094.

10. Esquela-Kerscher A, Slack FJ. Oncomirs - microRNAs with a role in cancer. Nat Rev Cancer 2006;6:259-269.

11. Rupaimoole R, Calin GA, Lopez-Berestein G, Sood AK. miRNA Deregulation in Cancer Cells and the Tumor Microenvironment. Cancer Discov 2016;6:235-246.

12. Hanahan D, Weinberg RA. Hallmarks of cancer: the next generation. Cell 2011;144:646-674.

13. Pencheva N, Tavazoie SF. Control of metastatic progression by microRNA regulatory networks. Nat Cell Biol 2013;15:546-554.

14. Zhang Y, Yang P, Wang XF. Microenvironmental regulation of cancer metastasis by miRNAs. Trends Cell Biol 2014;24:153-160.

15. Falcone G, Felsani A, D'Agnano I. Signaling by exosomal microRNAs in cancer. J Exp Clin Cancer Res 2015;34:32.

16. Hu M, Yao J, Cai L, Bachman KE, van den Brule F, Velculescu V, Polyak K. Distinct epigenetic changes in the stromal cells of breast cancers. Nat Genet 2005;37:899-905.

17. Tuhkanen H, Anttila M, Kosma VM, Heinonen S, Juhola M, Helisalmi S, Kataja V, et al. Frequent gene dosage alterations in stromal cells of epithelial ovarian carcinomas. Int J Cancer 2006;119:1345-1353.

18. Qiu W, Hu M, Sridhar A, Opeskin K, Fox S, Shipitsin M, Trivett M, et al. No evidence of clonal somatic genetic alterations in cancer-associated fibroblasts from human breast and ovarian carcinomas. Nat Genet 2008;40:650-655.

19. Kohlhapp FJ, Mitra AK, Lengyel E, Peter ME. MicroRNAs as mediators and communicators between cancer cells and the tumor microenvironment. Oncogene 2015;34:5857-5868.

20. Zhang D, Wang Y, Shi Z, Liu J, Sun P, Hou X, Zhang J, et al. Metabolic reprogramming of cancer-associated fibroblasts by IDH3alpha downregulation. Cell Rep 2015;10:1335-1348.

21. Penfornis P, Vallabhaneni KC, Whitt J, Pochampally R. Extracellular vesicles as carriers of microRNA, proteins and lipids in tumor microenvironment. Int J Cancer 2016;138:14-21.

22. Tominaga N, Katsuda T, Ochiya T. Micromanaging of tumor metastasis by extracellular vesicles. Semin Cell Dev Biol 2015;40:52-59.

23. Morello M, Minciacchi VR, de Candia P, Yang J, Posadas E, Kim H, Griffiths D, et al. Large oncosomes mediate intercellular transfer of functional microRNA. Cell Cycle 2013;12:3526-3536.

24. Josson S, Gururajan M, Sung SY, Hu P, Shao C, Zhau HE, Liu C, et al. Stromal fibroblast-derived miR-409 promotes epithelial-to-mesenchymal transition and prostate tumorigenesis. Oncogene 2015:34:2690-2699.

25. Doldi V, Callari M, Giannoni E, D'Aiuto F, Maffezzini M, Valdagni R, Chiarugi $\mathrm{P}$, et al. Integrated gene and miRNA expression analysis of prostate cancer associated fibroblasts supports a prominent role for interleukin-6 in fibroblast activation. Oncotarget 2015;6:31441-31460.

26. Madar S, Goldstein I, Rotter V. 'Cancer associated fibroblasts'--more than meets the eye. Trends Mol Med 2013;19:447-453.

27. Polanska UM, Orimo A. Carcinoma-associated fibroblasts: non-neoplastic tumour-promoting mesenchymal cells. J Cell Physiol 2013;228:1651-1657.

28. Sugimoto H, Mundel TM, Kieran MW, Kalluri R. Identification of fibroblast heterogeneity in the tumor microenvironment. Cancer Biol Ther 2006;5:1640-1646.

29. Mitra AK, Zillhardt M, Hua Y, Tiwari P, Murmann AE, Peter ME, Lengyel E. MicroRNAs reprogram normal fibroblasts into cancer-associated fibroblasts in ovarian cancer. Cancer Discov 2012;2:1100-1108.

30. Galon J, Mlecnik B, Bindea G, Angell HK, Berger A, Lagorce C, Lugli A, et al. Towards the introduction of the 'Immunoscore' in the classification of malignant tumours. J Pathol 2014·232:199-209.

31. Paulsson J, Micke P. Prognostic relevance of cancer-associated fibroblasts in human cancer. Semin Cancer Biol 2014;25:61-68.

32. Liao Y, Ni Y, He R, Liu W, Du J. Clinical implications of fibroblast activation protein-alpha in non-small cell lung cancer after curative resection: a new predictor for prognosis. J Cancer Res Clin Oncol 2013;139:1523-1528.

33. Ito M, Ishii G, Nagai K, Maeda R, Nakano Y, Ochiai A. Prognostic impact of cancer-associated stromal cells in patients with stage I lung adenocarcinoma. Chest 2012;142:151-158.

34. Ono S, Ishii G, Nagai K, Takuwa T, Yoshida J, Nishimura M, Hishida T, et al. Podoplanin-positive cancer-associated fibroblasts could have prognostic value independent of cancer cell phenotype in stage I lung squamous cell carcinoma: usefulness of combining analysis of both cancer cell phenotype and cancer-associated fibroblast phenotype. Chest 2013;143:963-970.

35. Ariga N, Sato E, Ohuchi N, Nagura H, Ohtani H. Stromal expression of fibroblast activation protein/seprase, a cell membrane serine proteinase and gelatinase, is associated with longer survival in patients with invasive ductal carcinoma of breast. Int J Cancer 2001:95:67-72

36. Schoppmann SF, Berghoff A, Dinhof C, Jakesz R, Gnant M, Dubsky P, Jesch B, et al. Podoplanin-expressing cancer-associated fibroblasts are associated with poor prognosis in invasive breast cancer. Breast Cancer Res Treat 2012;134:237-244.

37. Pula B, Jethon A, Piotrowska A, Gomulkiewicz A, Owczarek T, Calik J, Wojnar A, et al. Podoplanin expression by cancer-associated fibroblasts predicts poor outcome in invasive ductal breast carcinoma. Histopathology 2011;59:1249-1260

38. Li P, Shan JX, Chen XH, Zhang D, Su LP, Huang XY, Yu BQ, et al. Epigenetic silencing of microRNA-149 in cancer-associated fibroblasts mediates prostaglandin E2/interleukin-6 signaling in the tumor microenvironment. Cell Res 2015;25:588-603.

39. Taddei ML, Cavallini L, Comito G, Giannoni E, Folini M, Marini A, Gandellini $\mathrm{P}$, et al. Senescent stroma promotes prostate cancer progression: the role of miR-210. Mol Oncol 2014;8:1729-1746.

40. Al-Ansari MM, Aboussekhra A. miR-146b-5p mediates p16-dependent repression of IL- 6 and suppresses paracrine procarcinogenic effects of breast stromal fibroblasts. Oncotarget 2015;6:30006-30016.

41. De Wever O, Demetter P, Mareel M, Bracke M. Stromal myofibroblasts are drivers of invasive cancer growth. Int J Cancer 2008;123:2229-2238.

42. Clark JC, Thomas DM, Choong PF, Dass CR. RECK--a newly discovered inhibitor of metastasis with prognostic significance in multiple forms of cancer. Cancer Metastasis Rev 2007;26:675-683.

43. Bullock MD, Pickard KM, Nielsen BS, Sayan AE, Jenei V, Mellone M, Mitter R, et al. Pleiotropic actions of miR-21 highlight the critical role of deregulated stromal microRNAs during colorectal cancer progression. Cell Death Dis 2013;4:e684.

44. Au Yeung CL, Co NN, Tsuruga T, Yeung TL, Kwan SY, Leung CS, Li Y, et al. Exosomal transfer of stroma-derived miR21 confers paclitaxel resistance in ovarian cancer cells through targeting APAF1. Nat Commun 2016;7:11150.

45. Straussman R, Morikawa T, Shee K, Barzily-Rokni M, Qian ZR, Du J, Davis A, et al. Tumour micro-environment elicits innate resistance to RAF inhibitors through HGF secretion. Nature 2012;487:500-504.

46. Naito Y, Sakamoto N, Oue N, Yashiro M, Sentani K, Yanagihara K, Hirakawa $\mathrm{K}$, et al. MicroRNA-143 regulates collagen type III expression in stromal fibroblasts of scirrhous type gastric cancer. Cancer Sci 2014;105:228-235.

47. Acevedo VD, Ittmann M, Spencer DM. Paths of FGFR-driven tumorigenesis. Cell Cycle 2009;8:580-588

48. Konig A, Menzel T, Lynen S, Wrazel L, Rosen A, Al-Katib A, Raveche E, et al. Basic fibroblast growth factor (bFGF) upregulates the expression of bcl-2 in B cell chronic lymphocytic leukemia cell lines resulting in delaying apoptosis. Leukemia 1997;11:258-265.

49. Menzel T, Rahman Z, Calleja E, White K, Wilson EL, Wieder R, Gabrilove J. Elevated intracellular level of basic fibroblast growth factor correlates with stage of chronic lymphocytic leukemia and is associated with resistance to fludarabine. Blood 1996;87:1056-1063.

50. Sezer O, Jakob C, Eucker J, Niemoller K, Gatz F, Wernecke K, Possinger K. Serum levels of the angiogenic cytokines basic fibroblast growth factor (bFGF), vascular endothelial growth factor (VEGF) and hepatocyte growth factor (HGF) in multiple myeloma. Eur J Haematol 2001;66:83-88.

51. Song S, Wientjes MG, Gan Y, Au JL. Fibroblast growth factors: an epigenetic mechanism of broad spectrum resistance to anticancer drugs. Proc Natl Acad Sci U S A 2000;97:8658-8663.

52. Turner N, Pearson A, Sharpe R, Lambros M, Geyer F, Lopez-Garcia MA, Natrajan R, et al. FGFR1 amplification drives endocrine therapy resistance and is a therapeutic target in breast cancer. Cancer Res 2010;70:2085-2094.

53. Musumeci M, Coppola V, Addario A, Patrizii M, Maugeri-Sacca M, Memeo L, Colarossi $\mathrm{C}$, et al. Control of tumor and microenvironment cross-talk by miR-15a and miR-16 in prostate cancer. Oncogene 2011;30:4231-4242.

54. Bronisz A, Godlewski J, Wallace JA, Merchant AS, Nowicki MO, Mathsyaraja $\mathrm{H}$, Srinivasan $\mathrm{R}$, et al. Reprogramming of the tumour microenvironment by stromal PTEN-regulated miR-320. Nat Cell Biol 2012;14:159-167.

55. Trimboli AJ, Cantemir-Stone CZ, Li F, Wallace JA, Merchant A, Creasap N, Thompson JC, et al. Pten in stromal fibroblasts suppresses mammary epithelial tumours. Nature 2009:461:1084-1091.

56. Vivacqua A, De Marco P, Santolla MF, Cirillo F, Pellegrino M, Panno ML, Abonante S, et al. Estrogenic gper signaling regulates mir144 expression in cancer cells and cancer-associated fibroblasts (cafs). Oncotarget 2015;6:16573-16587.

57. Yasuda K, Torigoe T, Mariya T, Asano T, Kuroda T, Matsuzaki J, Ikeda K, et al. Fibroblasts induce expression of FGF4 in ovarian cancer stem-like cells/cancer-initiating cells and upregulate their tumor initiation capacity. Lab Invest 2014;94:1355-1369.

58. Hasegawa T, Yashiro M, Nishii T, Matsuoka J, Fuyuhiro $\mathrm{Y}$, Morisaki T, Fukuoka $\mathrm{T}$, et al. Cancer-associated fibroblasts might sustain the stemness of scirrhous gastric cancer cells via transforming growth factor-beta signaling. Int J Cancer 2014;134:1785-1795.

59. Weiland A, Roswall P, Hatzihristidis TC, Pietras K, Ostman A Strell C. Fibroblast-dependent regulation of the stem cell properties of cancer cells. Neoplasma 2012;59:719-727.

60. A. The tumour-induced systemic environment as a critical regulator of cancer progression and metastasis. Nat Cell Biol 2014;16:717-727.

61. Roy A, Bera S. CAF cellular glycolysis: linking cancer cells with the microenvironment. Tumour Biol 2016.

62. Fang $R$, Xiao $T$, Fang $Z$, Sun $Y$, Li F, Gao $Y$, Feng $Y$, et al. MicroRNA-143 (miR-143) regulates cancer glycolysis via targeting hexokinase 2 gene. J Biol Chem 2012;287:23227-23235.

63. Peschiaroli A, Giacobbe A, Formosa A, Markert EK, Bongiorno-Borbone L, Levine AJ, Candi E, et al. miR-143 regulates hexokinase 2 expression in cancer cells. Oncogene 2013;32:797-802. 
64. Hu JW, Sun P, Zhang DX, Xiong WJ, Mi J. Hexokinase 2 regulates G1/S checkpoint through CDK2 in cancer-associated fibroblasts. Cell Signal 2014;26:2210-2216.

65. Hu Y, Yan C, Mu L, Huang K, Li X, Tao D, Wu Y, et al. Fibroblast-Derived Exosomes Contribute to Chemoresistance through Priming Cancer Stem Cells in Colorectal Cancer. PLoS One 2015;10:e0125625.

66. Tanaka K, Miyata H, Sugimura K, Fukuda S, Kanemura T, Yamashita K, Miyazaki $Y$, et al. miR-27 is associated with chemoresistance in esophageal cancer through transformation of normal fibroblasts to cancer-associated fibroblasts. Carcinogenesis 2015;36:894-903.

67. Donahue TR, Nguyen AH, Moughan J, Li L, Tatishchev S, Toste P, Farrell JJ. Stromal microRNA-21 levels predict response to 5-fluorouracil in patients with pancreatic cancer. J Surg Oncol 2014;110:952-959.

68. Li J, Guan J, Long X, Wang Y, Xiang X. mir-1-mediated paracrine effect of cancer-associated fibroblasts on lung cancer cell proliferation and chemoresistance. Oncol Rep 2016;35:3523-3531.

69. Pang W, Su J, Wang Y, Feng H, Dai X, Yuan Y, Chen X, et al. Pancreatic cancer-secreted miR-155 implicates in the conversion from normal fibroblasts to cancer-associated fibroblasts. Cancer Sci 2015;106:1362-1369.

70. Naito Y, Yasuno K, Tagawa H, Sakamoto N, Oue N, Yashiro M, Sentani K, et al. MicroRNA-145 is a potential prognostic factor of scirrhous type gastric cancer. Oncol Rep 2014;32:1720-1726.

71. Nouraee N, Van Roosbroeck K, Vasei M, Semnani S, Samaei NM, Naghshvar $\mathrm{F}$, Omidi AA, et al. Expression, tissue distribution and function of miR-21 in esophageal squamous cell carcinoma. PLoS One 2013;8:e73009.

72. Yang TS, Yang XH, Chen X, Wang XD, Hua J, Zhou DL, Zhou B, et al. MicroRNA-106b in cancer-associated fibroblasts from gastric cancer promotes cell migration and invasion by targeting PTEN. FEBS Lett 2014;588:2162-2169. 